\title{
HBIM : une analyse de la littérature scientifique
}

\section{HBIM: A scientific literature analysis}

\author{
Alexandra Stoleru et Kévin Jacquot* \\ MAP-Aria (UMR 3495 CNRS/MC), ENSA Lyon, France
}

\begin{abstract}
Résumé. Dans le sillage de la lente diffusion du BIM dans les milieux professionnels de la construction, un nombre croissant d'expérimentations sont menées dans le domaine de l'existant à forte valeur patrimoniale, principalement dans le monde de la recherche. Cette communication étudie l'évolution de la production scientifique pour dégager des tendances et identifier les futurs axes de développement de cette approche relativement nouvelle et encore mal définie.
\end{abstract}

Mots-clés. HBIM, BIM, patrimoine culturel, bibliométrie, scientométrie.

\begin{abstract}
In the wake of the slow spread of BIM in professional construction circles, a growing number of experiments are being conducted in the field of existing buildings with high heritage value, mainly in the world of research. This paper examines the evolution of scientific production in order to identify trends and the future development of this relatively new and as yet still undefined approach.
\end{abstract}

Keywords. HBIM, BIM, cultural heritage, bibliometry, scientometry.

\section{Introduction}

De plus en plus utilisé dans le domaine de la construction, le Building Information Modeling (BIM) apparaît aujourd'hui comme une approche incontournable, mais qui peine à s'installer en France (Hochscheid \& Halin, 2018). L'application de ce paradigme au contexte patrimonial est cependant plus récente. Connu sous le terme de Heritage ou encore Historic Building Modeling, le HBIM est défini pour la première fois par Murphy et al. (2009) comme " un nouveau système pour la modélisation des structures historiques ». Or, cette définition met l'accent uniquement sur les objets au détriment des processus collaboratifs, pourtant un enjeu inédit. Car bien que BIM et HBIM partagent certains acteurs, processus et objectifs, le patrimoine bâti a ses propres spécificités relevant des sciences du patrimoine, domaine de recherche interdisciplinaire associant sciences sociales,

${ }^{*}$ Corresponding author: kevin.jacquot@map.cnrs.fr 
sciences expérimentales et sciences du numérique agissant de concert pour améliorer la connaissance, la conservation, la restauration et la transmission du patrimoine.

La dimension transdisciplinaire qu'exige l'étude du patrimoine ne peut qu'interroger l'aspect collaboratif et l'hétérogénéité des données produites par les différents corps de métiers et soulève la question de la gestion des connaissances autour d'une maquette numérique centrale (d'Avezac de Castera, 2018). Ainsi, outre les aspects géométriques liés à la modélisation de l'existant («as-built»), les dimensions liées à la coordination et la collaboration, la gestion du cycle de vie du bâtiment, ou encore les simulations virtuelles, les éléments propres à l'approche BIM sont particulièrement intéressants dans un contexte d'analyse, d'exploitation ou de valorisation du patrimoine bâti. Cependant, de nombreuses publications sur le HBIM semblent aborder le sujet par une approche qualitative et manuelle limitée au traitement géométrique. Plusieurs travaux de référence, à l'image des états de l'art de López et al. (2018), Bruno et al. (2018) ou encore García et al. (2018) tendent à confirmer cet état de fait.

Nous proposons donc à travers cet article un état de l'art reposant sur une méthodologie originale au regard des publications susmentionnées, c'est-à-dire par une approche bibliométrique et scientométrique intégrée afin de cartographier de manière quantitative l'ensemble des domaines de connaissances autour desquels se construit le HBIM. L'objectif est d'étudier l'évolution de la production scientifique, dégager des tendances et identifier les potentiels axes de développement de cette approche relativement nouvelle et encore mal définie. Après avoir présenté la méthodologie adoptée, nous nous attacherons à présenter les résultats obtenus et enfin amorcer une discussion autour des thématiques identifiées. Conscients de la complexité d'une telle évaluation, notre étude ne prétend pas à l'exhaustivité, mais soulève des questions quant à l'évolution de la méthodologie HBIM.

\section{Méthodologie}

La présente publication se construit en trois temps : une analyse bibliométrique de la littérature scientifique du domaine, suivie par une analyse scientométrique afin de comprendre le développement de cette pratique et enfin une discussion autour des résultats obtenus corroborés par une analyse manuelle plus approfondie. Une approche bibliométrique s'impose étant donnée la quantité de publications existantes autour du HBIM.

\subsection{Analyse bibliométrique}

La première étape de cette étude réside en une analyse bibliométrique dont la validité réside principalement dans la représentativité de la base de données au sein du domaine étudié (Mongeon \& Paul-Hus, 2016). C'est la raison pour laquelle nous avons utilisé Scopus, base de données élaborée par l'éditeur Elsevier. Celle-ci répertorie un nombre plus important de revues et des publications scientifiques plus récentes (en comparaison avec des bases de données notoires comme WOS), notamment dans des champs multidisciplinaires (Aghaei Chadegani et al., 2013).

Une recherche par mots-clés et opérateurs logiques a été réalisée dans un premier temps. L'expression choisie afin de couvrir les articles du domaine n'utilisant pas forcément le mot « HBIM » tout en réduisant le nombre des articles hors sujet (chimie organique, etc.) est la suivante : ('hbim' AND ('heritage' OR 'historic' OR 'cultural')) OR ('bim' AND ('heritage' OR 'historic' OR 'cultural')). Un nombre de 619 contributions incluant ces mots-clés dans le titre, le résumé ou les mots-clés indexés a été identifié dans un premier temps.

Les résultats sont par la suite limités aux articles de revues, contributions passées par un processus rigoureux d'évaluation par les pairs. Sont par la suite écartés les articles rédigés 
dans une autre langue que l'anglais, aucun article des résultats n'étant en français. Suite à cette étape réalisée grâce aux filtres proposés par Scopus, 155 résultats sont retenus. Une étape de filtrage manuel nous a par la suite permis d'écarter sept faux positifs ${ }^{2}$ et de ne conserver que les articles considérés pertinents, car concernant le patrimoine bâti ${ }^{3}$. Le corpus final compte ainsi 98 articles scientifiques publiés entre 2008 et 2019.

\subsection{Analyse scientométrique}

La visualisation d'un domaine peut s'avérer indispensable à son analyse, en particulier dans les domaines de connaissances multidisciplinaires et en évolution rapide (Börner et al., 2003). Le logiciel VOSViewer (Van Eck \& Waltman, 2010, 2017) a été choisi pour l'analyse scientométrique. Il permet de cartographier et visualiser ces métadonnées sous la forme de graphes (figures 2-5) à partir des données bibliométriques des articles précédemment sélectionnés (auteurs, résumés, mots-clés, année de publication, références, etc.) exportées depuis Scopus. Si la taille des nœuds représente le nombre d'occurrences du terme étudié, la distance entre les différents nœuds est quant à elle une indication de proximité de la relation entre deux termes (Van Eck \& Waltman, 2014). Les relations entre les revues, publications, mots-clés, ou encore termes extraits par fouilles de textes des titres et résumés des articles du corpus ont pu ainsi être analysées.

\section{Résultats}

La répartition annuelle des articles, le couplage bibliographique des revues dans lesquelles ceux-ci sont publiés, leurs citations, l'occurrence des mots-clés des auteurs et enfin celle les termes extraits par fouille de texte sont les aspects étudiés et présentés dans cette partie.

\subsection{Répartition annuelle des publications}

Nous nous sommes intéressés dans un premier temps à la répartition des articles selon les années de publication. La figure 1 traduit ainsi une croissance notable du nombre de publications dans le domaine du HBIM. Un pic de publications peut être constaté en 2016 : 12 publications parmi les 17 publiées cette année-là étant des articles de la revue Disegnarecon, notamment dans un numéro autour des dimensions du $\mathrm{BIM}^{4}$. Une accélération de cette croissance est visible notamment depuis 2016, le nombre d'articles publiés durant l'année 2019 s'élevant à $33^{5}$, témoignant effectivement d'un intérêt croissant pour le domaine.

\footnotetext{
${ }^{2}$ L'acronyme « BIM » a également été rencontré dans des domaines comme la chimie générant des résultats « faux positifs».

${ }^{3}$ Certains articles traitant de la question du BIM et dont les termes «heritage » ou «cultural » sont employés dans un contexte général dans le résumé, ne concernent pas le patrimoine bâti.

${ }^{4}$ Dix articles sont publiés dans Disegnarecon 2016. Vol 9, $\mathrm{n}^{\circ} 16$ : B.I.M Dimensions.

${ }^{5}$ Il s'agit d'un nombre incomplet, l'étude ne recouvrant pas le mois de décembre de l'année en cours.
} 


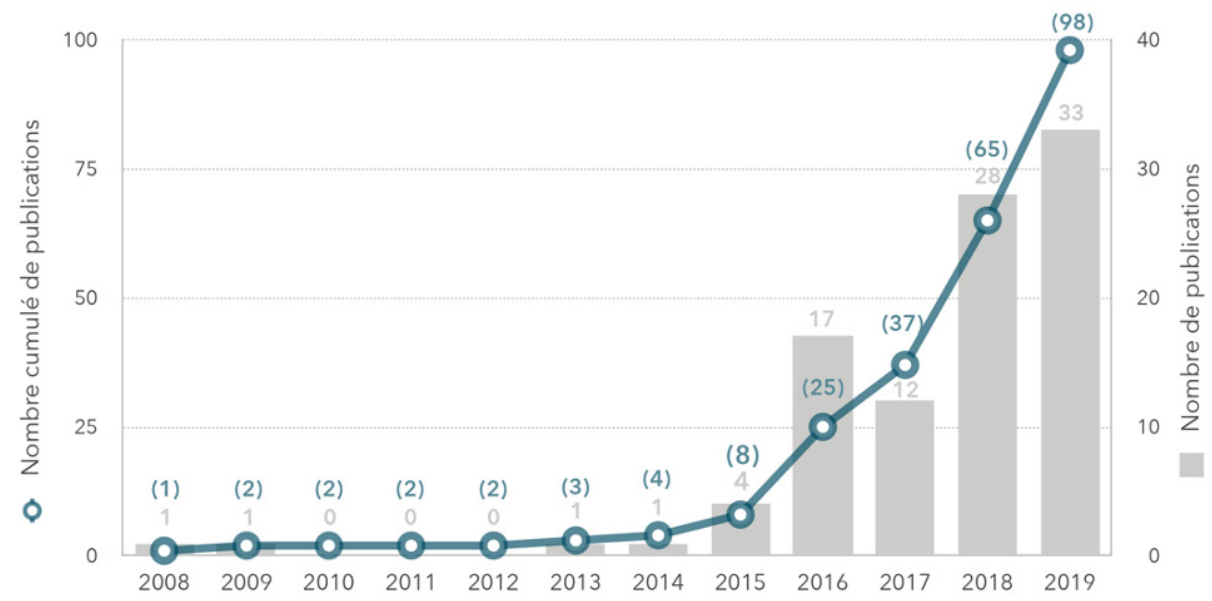

Figure 1. Répartition annuelle des 98 articles publiés de 2008 à 2019 dans le domaine du HBIM résultant de la méthodologie adoptée

\subsection{Analyse du couplage bibliographique des revues}

Les revues ayant publié les articles sélectionnés sont par la suite analysées par la cartographie de leur couplage bibliographique (figure 2). Pour cela, un seuil correspondant au minimum à une citation reçue par les publications d'une revue est établi. Sur l'ensemble des 45 revues, 30 franchissent ce seuil. La taille des nœuds est plus ou moins importante selon le nombre d'articles publiés dans une revue. En ce qui concerne la proximité entre deux nœuds, celle-ci est définie par le nombre de références que les articles de deux revues ont en commun. Ainsi, plus le nombre des références communes est important, plus deux cercles seront proches. Enfin, la couleur des nœuds renvoie à l'année moyenne des articles publiés par la revue.

Excentrée dans le graphe se trouve la revue Structural Survey, dans laquelle est publié le premier article définissant le terme HBIM (Murphy et al., 2009). Avec un total de 16 articles, la revue Disegnarecon a publié le nombre le plus important d'articles dans le domaine. Nous nous interrogeons cependant sur la portée de ces publications, le CiteScore ${ }^{6}$ de la revue étant relativement faible $(0,13$ alors que le CiteScore moyen des revues sélectionnées est de 1,7). C'est enfin International Journal of Architectural Heritage qui présente un nombre relativement important de publications récentes, avec six articles publiés en 2019 rapportés à la moyenne pour cette année qui est de 1,3 publication par revue.

Ce graphe traduit enfin une démultiplication relativement récente du nombre de revues dans lesquelles sont publiés des articles dans le domaine du HBIM. Une légère diversification des orientations de ces revues est également à noter. Celle-ci peut en effet traduire une adoption des méthodologies HBIM dans des champs disciplinaires plus variés.

\footnotetext{
${ }^{6}$ Le CiteScore est un indicateur de notoriété crée par Elsevier qui correspond au ratio, calculé sur une période de trois ans, entre le nombre de citations reçues et le nombre d'articles publié par une revue.
} 


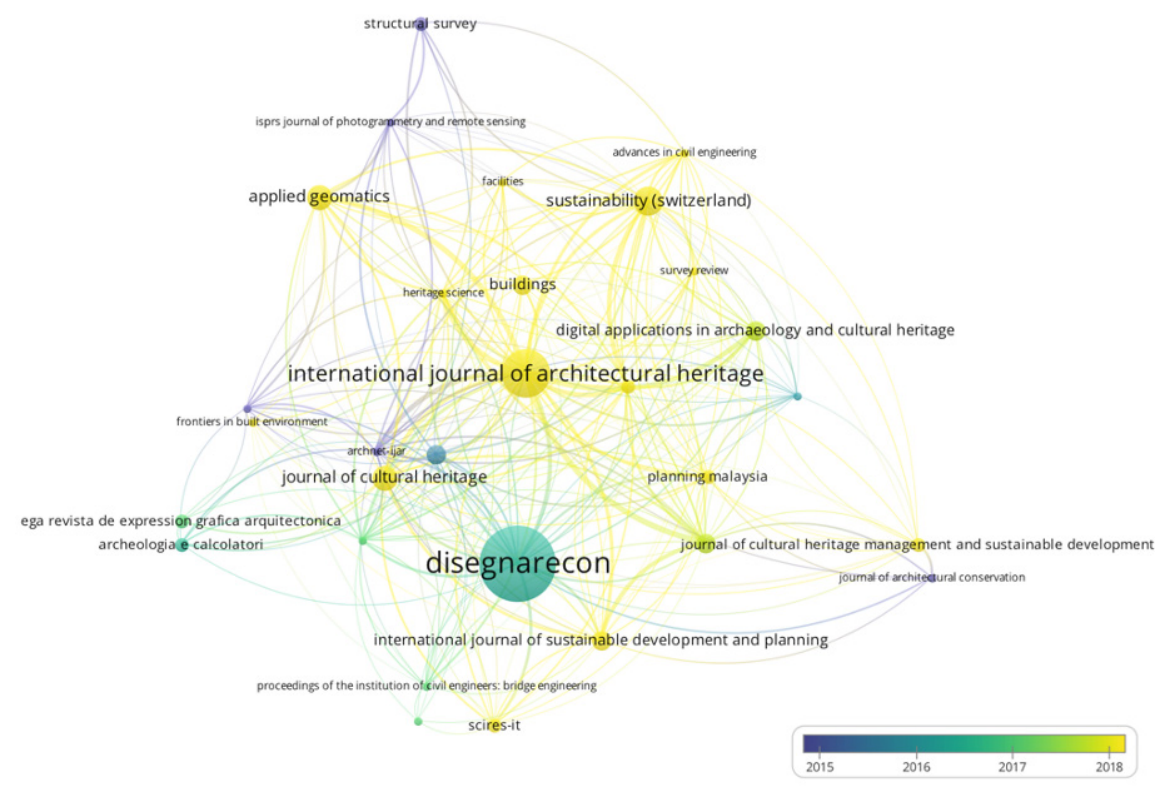

Figure 2. Graphe du couplage bibliographique des revues ayant publié les articles identifiés

\subsection{Analyse des articles}

Nous nous sommes par la suite intéressés aux contributions abordant la question du HBIM. La figure 3 illustre ainsi les relations des publications en fonction de leurs citations. Parmi les 98 publications du corpus, seuls les 61 articles citant au moins un autre article du corpus ont été sélectionnés pour réaliser ce graphe. Ainsi, parmi les publications les plus citées, susceptibles donc d'avoir un impact plus important dans le domaine, figurent Murphy et al. (2013) avec 158 citations suivie par Murphy et al. (2009) avec 115 citations.

Ces deux articles apparaissent par ailleurs comme étant des références lorsque l'on parle de HBIM. Cela peut effectivement être expliqué par le fait qu'il s'agisse des auteurs qui sont à l'origine du terme «HBIM », et dont la définition a été reprise par la suite par les travaux abordant ce domaine en cours de construction.

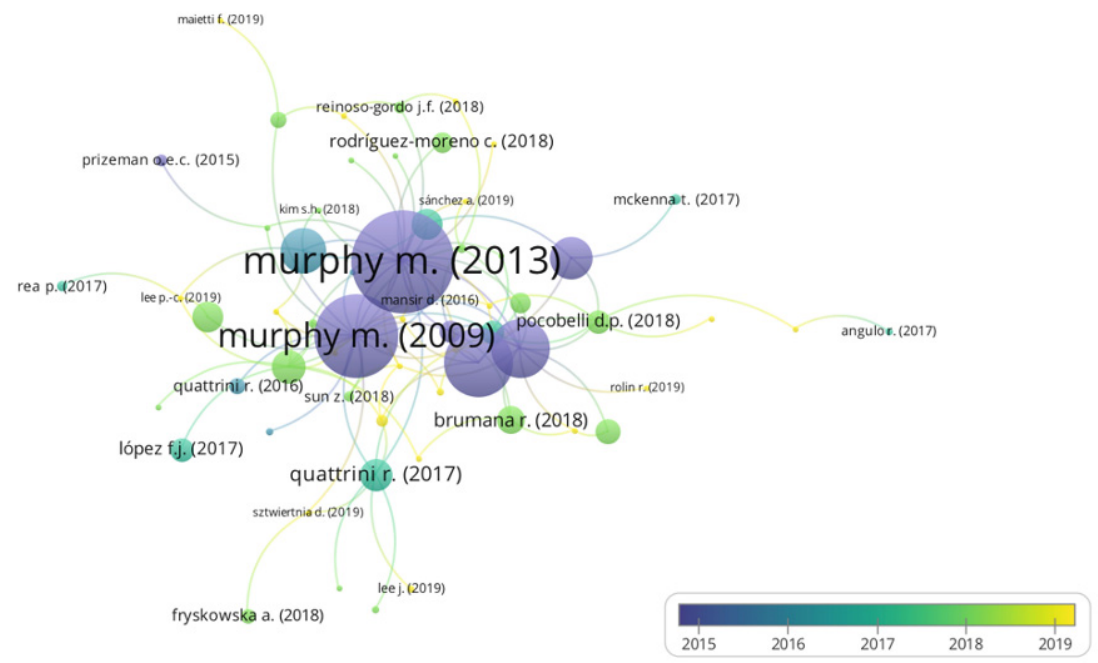

Figure 3. Graphe des citations des articles citant au moins un autre article du corpus 


\subsection{Analyse de l'occurrence des mots-clés}

Les mots-clés, définis par les auteurs, traduisent les points d'intérêt des études menées et présentées dans la littérature scientifique (Lee \& Su, 2010). La taille des nœuds est plus importante lorsque le terme présente plus d'occurrences. Un seuil de deux occurrences a été établi pour la création du graphe (figure 4) et 39 termes sur les 330 ont été analysés. Par ailleurs, un thésaurus nous a permis d'harmoniser les différentes variations des termes (synonymes, anglais britannique et américain, etc.).

Parmi les mots-clés les plus utilisés figurent les termes « hbim» avec 43 occurrences et «bim» avec 38 occurrences. Des termes relatifs au domaine d'application, comme «cultural heritage» ou "architectural heritage» figurent également parmi les plus utilisés. Des termes généralement liés aux aspects géométriques de la modélisation

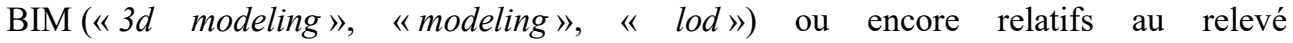
numérique («digital survey», «terrestrial laser scanning», "photogrammetry» ou "point cloud») sont également fréquemment employés. Parmi les termes moins utilisés figurent des mots-clés comme («virtual heritage», «gis» ou encore "classification system »).

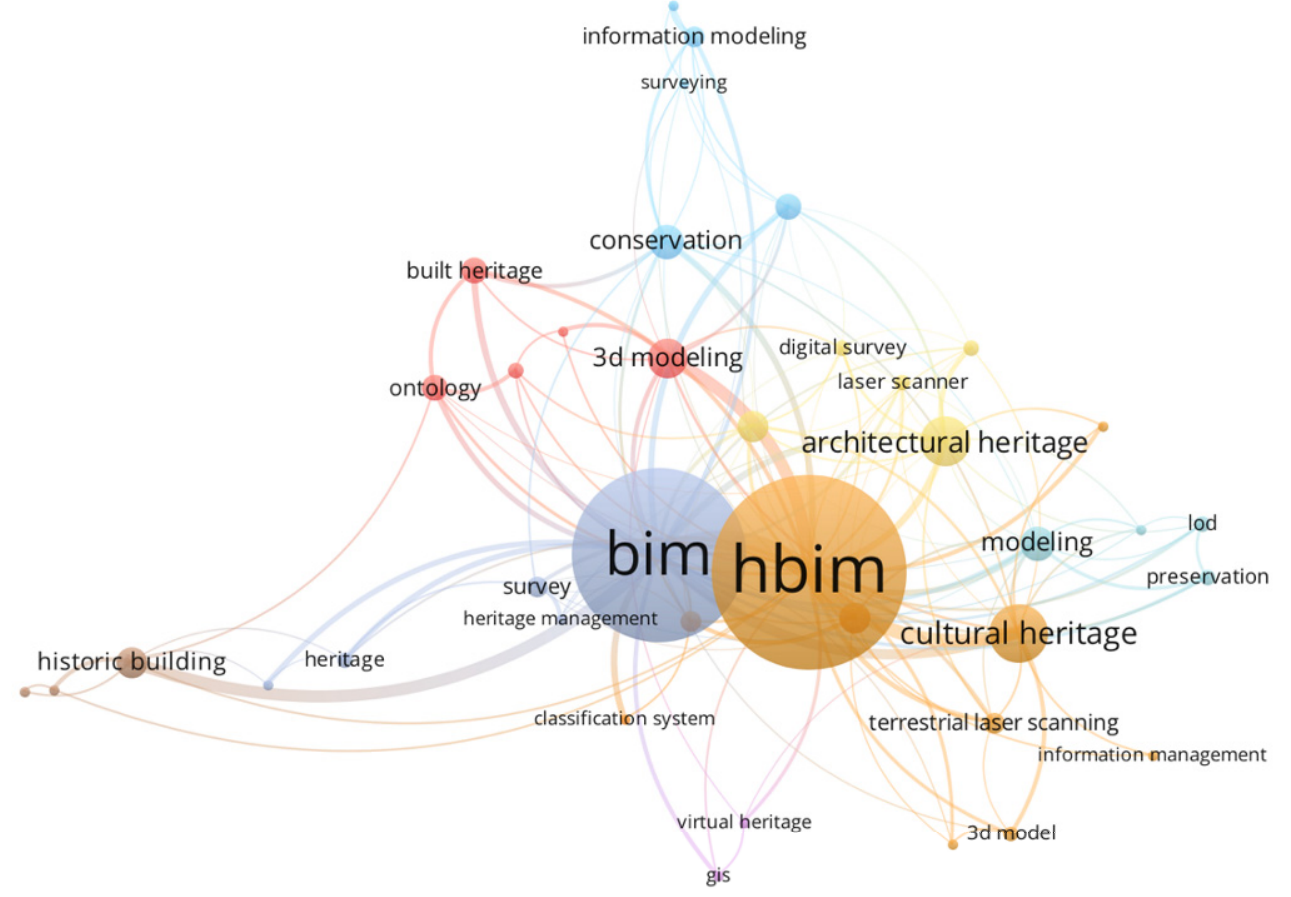

Figure 4. Graphe des co-occurrences des mots-clés utilisés par les auteurs

\subsection{Analyse des termes extraits par fouille de texte}

Nous avons enfin cartographié les termes récurrents en utilisant la fonctionnalité de fouille de texte de VOSviewer qui s'appuie sur la suite OpenNLP d'Apache (Van Eck \& Waltman, 2014). L'analyse a été réalisée sur les titres et résumés des articles scientifiques sélectionnés. Les termes de "faible pertinence» (ou de signification générale) ont une distribution plus ou moins égale de leurs co-occurrences, tandis que les termes de « haute pertinence » (ou de signification spécifique) ont une distribution de leurs co-occurrences qui est significativement biaisée vers certaines phrases nominales (Van Eck \& Waltman, 
2014). Un seuil de dix occurrences a été établi, sur les 47 termes dépassant ce seuil, seuls les 28 premiers (soit $60 \%$ ) les plus pertinents ont été sélectionnés ${ }^{7}$.

Les termes sont par la suite regroupés dans des familles appelées clusters. Deux clusters distincts sont ainsi mis en évidence (figure 5), correspondant à deux champs de connaissances du domaine. Le premier (orange - gauche) regroupe des termes relatifs notamment à l'application de la méthodologie dans un contexte patrimonial comme " heritage », " maintenance ». " restoration», "preservation », "cultural heritage » et " architectural heritage ». Le deuxième (bleu - droite) comporte des termes relatifs aux aspects géométriques d'une maquette numérique BIM comme "geometry», « object», «detail» ou encore « $3 d$ model ». Nous remarquons enfin que, contrairement à d'autres dimensions BIM, la modélisation géométrique est effectivement surreprésentée.

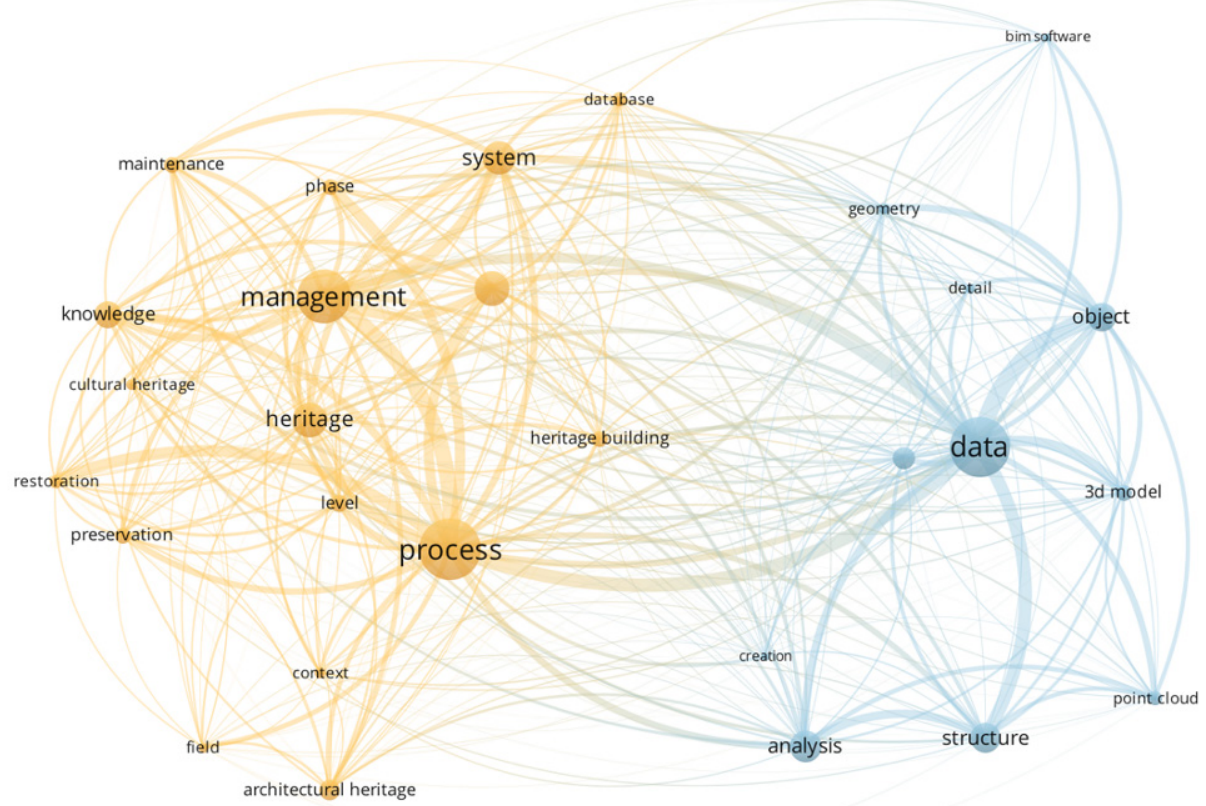

Figure 5. Graphe des co-occurrences de termes extraits des titres et résumés des articles par fouille de texte

\section{Discussion}

À la différence de la plupart des états de l'art sur le sujet, notre approche cherche à mettre en œuvre une analyse statistique et automatisée : une cartographie bibliométrique et scientométrique intégrée des recherches sur le HBIM. Nos résultats montrent une augmentation du nombre de revues dans lesquels la notion de HBIM est présente (3.2), une référence systématisée à la publication originelle de Murphy et al. (2009) (3.3), des problématiques empreintes d'un lexique spécifique aux enjeux de l'acquisition et du traitement géométrique tant dans les mots-clés (3.4) que dans les corps de texte (3.5).

Suite à la présentation des résultats bibliométriques et scientométriques du corpus de publications scientifiques étudié, nous tenterons à présent d'aborder les différentes thématiques issues d'une analyse plus approfondie de la littérature scientifique. Il s'agit bien évidemment d'une étape manuelle et chronophage, encore en cours de déroulement.

${ }^{7}$ Cette méthode est conseillée par van Eck, Waltman (2014) et s'appuie sur un score de pertinence calculé pour chaque terme. 


\subsection{Représentation des dimensions géométriques}

Un des axes de recherche majeurs autour duquel se concentre une partie conséquente de la littérature scientifique réside dans la représentation de l'existant. Le patrimoine bâti, plus encore que l'architecture contemporaine, peut-être qualifié de non-standard. Il est riche d'un vocabulaire formel multiséculaire, vernaculaire qui, de surcroît, a souvent subi des altérations, parachevant la singularisation des objets étudiés qui sont alors difficilement modélisables à l'aide des logiciels utilisés couramment dans le BIM.

Plusieurs solutions se dessinent aux enjeux de modélisation dans un contexte BIM, à partir d'un relevé numérique de l'existant suivi par une étape faisant appel à des techniques de retopologie ou de rétroconception. Une première réside dans la création de bibliothèques d'objets paramétriques, s'appuyant par ailleurs sur une documentation historique (Baik, 2017 ; Dore \& Murphy, 2014). Cependant, cette approche reste très peu généralisable et ne peut être exhaustive, visant souvent une période et un style architectural bien précis. Une deuxième approche réside dans l'automatisation des opérations de transformation de nuages de points issus de relevés numériques en données compatibles avec un environnement BIM. Cette opération demeure néanmoins largement manuelle.

Outre la perte d'informations issue de la première approche, les deux tentent principalement de réduire les imprécisions d'une modélisation dont les outils ne sont pas adaptés aux enjeux de la représentation du patrimoine bâti.

Nous pouvons effectivement formuler l'hypothèse d'un développement plus important de cet axe de recherche qui s'explique notamment par l'existence de travaux de rétroconception largement antérieurs à l'apparition du HBIM, qui se traduit par une littérature scientifique abondante, à partir de la formalisation d'une première définition de Murphy et al. (2009), largement orientée vers ces aspects géométriques susmentionnés.

\subsection{Représentation des aspects sémantiques, gestion des connaissances et exploitation de la maquette numérique}

Si l'intérêt d'un environnement BIM, réside non seulement dans la représentation géométrique, mais aussi dans les relations entre les objets modélisés et leurs attributs, les connaissances historiques et patrimoniales ne sont pas à négliger. Bien que la représentation géométrique d'un objet patrimonial complexe soit primordiale en vue de sa compréhension, les aspects sémantiques liés à la documentation historique, aux connaissances (techniques constructives, matériaux, etc.) ne peuvent être négligés dans le contexte de l'application du BIM au patrimoine bâti. Simeone et al. (2019) proposent dans ce sens un enrichissement sémantique de la méthodologie BIM à travers les technologies du Web Sémantique et plus particulièrement grâce à l'implémentation d'une ontologie dans le but d'améliorer la représentation et la gestion des connaissances dans le contexte patrimonial.

Les données sémantiques, les attributs d'objets et leurs relations sont primordiaux pour l'exploitation d'une maquette numérique. Véritable support pour la prise décisionnelle, celle-ci est indispensable pour une gestion du patrimoine tout au long de son cycle de vie.

Que cela soit dans le cadre de projets de réhabilitation ou de conservation, la maquette numérique devient un support pour des analyses de structure (Barazzetti et al., 2015 ; Rolin et al., 2019), des simulations énergétiques (Kim, 2018) ou encore des opérations de conservation planifiée (Bruno \& Roncella, 2019).

\subsection{Aspects collaboratifs}

Bien que la question de l'adoption du BIM de manière générale soit largement étudiée, les acteurs et les pratiques du patrimoine ne sont pas les mêmes que ceux du secteur de la 
construction. La question de l'adoption de cette méthodologie par les corps de métiers concernés serait effectivement intéressante à aborder. De surcroît, l'aspect collaboratif demeure très peu abordé dans le contexte du HBIM, alors que la diversité des acteurs (encore plus importante en raison de la nécessité d'un regard multidisciplinaire en vue d'une compréhension généralisée d'un objet patrimonial complexe) n'est pas à négliger. La question de la collaboration de différents corps de métiers pouvant être amenés à intervenir sur le patrimoine ou encore de l'interopérabilité des données hétérogènes produites mériterait d'être étudiée.

\section{Conclusion}

Face à une littérature scientifique croissante dans le domaine du HBIM, nous avons proposé dans cet article les résultats de l'analyse associant approches bibliométrique et scientométrique, corroborés par une discussion se basant sur une étude qualitative visant à identifier les points d'intérêts du domaine, mais aussi de mettre en lumière des champs de connaissances moins explorés au regard de ceux du BIM dont le HBIM est dérivé.

Les premiers résultats de cette étude mettent en évidence une concentration de la littérature scientifique autour des enjeux géométriques que soulèvent une modélisation d'un patrimoine bâti existant dans un environnement BIM. Bien que certains travaux commencent à s'intéresser à la question, des approches basées sur une ontologie afin d'enrichir sémantiquement la maquette numérique restent à être développées. Les données sémantiques enrichissant la maquette numérique sont par ailleurs indispensables à son exploitation et à la gestion finalement d'un patrimoine bâti tout au long de son cycle de vie. Enfin, l'absence de littérature relative à l'aspect collaboratif propre à un environnement BIM démontre bien qu'il s'agit d'un élément qui reste à questionner dans le domaine patrimonial.

Enfin, un certain nombre de limites de cette approche peuvent effectivement être formulées. Outre les choix faits concernant les étapes de filtrages du corpus, un des reproches pouvant être fait concerne la reproductibilité des procédures présentées. Il serait par ailleurs intéressant d'élargir cette étude à l'ensemble des contributions scientifiques (articles de colloques, livres, etc.), afin de comparer les résultats. De plus, nous nous interrogeons sur la pertinence de certains termes présents dans les résumés, leur emploi dans un contexte général ne pouvant pas garantir qu'il s'agit d'un élément réellement exploité dans l'article. Élargir la fouille de texte aux corps des articles nous permettrait ainsi de surmonter ce biais et confirmer ou infirmer les résultats par une analyse plus poussée.

Afin de pallier les limites d'une approche quantitative et qualitative de la littérature scientifique, il faudrait interroger également les projets mettant en œuvre des méthodologies HBIM. Cela nous permettrait de mettre en perspective la faisabilité du HBIM, les conditions devant être remplies afin de faire collaborer les professionnels de la construction, les professionnels de l'intervention sur le bâtiment et sites anciens, ainsi que les différentes communautés scientifiques faisant du patrimoine leur objet d'étude.

\section{Bibliographie}

Aghaei Chadegani, A., Salehi, H., Md Yunus, M. M., Farhadi, H., Fooladi, M., Farhadi, M., \& Ale Ebrahim, N. (2013). A comparison between two main academic literature collections : Web of science and scopus databases. Asian Social Science, 9(5), 18-26.

Baik, A. (2017). From point cloud to Jeddah Heritage BIM Nasif Historical House - case study. Digital Applications in Archaeology and Cultural Heritage, 4, 1-18. 
Barazzetti, L., Banfi, F., Brumana, R., Gusmeroli, G., Previtali, M., \& Schiantarelli, G. (2015). Cloud-to-BIM-to-FEM : Structural simulation with accurate historic BIM from laser scans. Simulation Modelling Practice and Theory, Complete (57), 71-87.

Börner, K., Chen, C., \& Boyack, K. W. (2003). Visualizing knowledge domains. Annual Review of Information Science and Technology, 37(1), 179-255.

Bruno, N., \& Roncella, R. (2019). HBIM for conservation : A new proposal for information modeling. Remote Sensing, 11(15).

Bruno, S., De Fino, M., \& Fatiguso, F. (2018). Historic Building Information Modelling: Performance assessment for diagnosis-aided information modelling and management. Automation in Construction, 86, 256-276.

d'Avezac de Castera, L. (2018). Atouts et enjeux du BIM pour les travaux dans l'existant à forte valeur patrimoniale. SFA - Le Bulletin, (54), 26-29.

Dore, C., \& Murphy, M. (2014). Semi-automatic generation of as-built BIM façade geometry from laser and image data. Journal of Information Technology in Construction, 19, 20-46.

García, E. S., García-Valldecabres, J., \& Blasco, M. J. V. (2018). The Use Of Hbim Models As A Tool For Dissemination And Public Use Management Of Historical Architecture : A Review. International Journal of Sustainable Development and Planning, 13(1), 96107.

Hochscheid, É., \& Halin, G. (2018). L'adoption du BIM dans les agences d'architecture en France. SHS Web of Conferences, 47, 01009.

Kim, S. H. (2018). Assessing the needs and gaps of building information technologies for energy retrofit of historic buildings in the Korean context. Sustainability (Switzerland), $10(5)$.

Lee, P.-C., \& Su, H.-N. (2010). Investigating the Structure of Regional Innovation System Research through Keyword Co-occurrence and Social Network Analysis.

López, F. J., Lerones, P. M., Llamas, J., Gómez-García-Bermejo, J., \& Zalama, E. (2018). A Review of Heritage Building Information Modeling (H-BIM). Multimodal Technologies and Interaction, 2(2), 21.

Mongeon, P., \& Paul-Hus, A. (2016). The Journal Coverage of Web of Science and Scopus : A Comparative Analysis. Scientometrics, 106(1), 213-228.

Murphy, M., Mcgovern, E., \& Pavia, S. (2009). Historic building information modelling (HBIM). Structural Survey, 27(4), 311-327.

Murphy, M., McGovern, E., \& Pavia, S. (2013). Historic Building Information Modelling Adding intelligence to laser and image based surveys of European classical architecture. ISPRS Journal of Photogrammetry and Remote Sensing, 76, 89-102.

Rolin, R., Antaluca, E., Batoz, J.-L., Lamarque, F., \& Lejeune, M. (2019). From point cloud data to structural analysis through a geometrical hBIM-oriented model. Journal on Computing and Cultural Heritage, 12(2).

Simeone, D., Cursi, S., \& Acierno, M. (2019). BIM semantic-enrichment for built heritage representation. Automation in Construction, 97, 122-137.

Van Eck, N. J., \& Waltman, L. (2010). Software survey: VOSviewer, a computer program for bibliometric mapping. Scientometrics, 84(2), 523-538.

Van Eck, N. J., \& Waltman, L. (2014). Visualizing Bibliometric Networks. Dans Y. Ding, R. Rousseau, \& D. Wolfram (Éds), Measuring Scholarly Impact : Methods and Practice (pp. 285-320). Cham : Springer International Publishing.

Van Eck, N. J., \& Waltman, L. (2017). VOSviewer Manual. Manual for VOSviewer version 1.6.6. 